\title{
Multifunctional Gratings for Multiband Spatial Filtering, Retroreflection, Splitting and Demultiplexing Based on C2 Symmetric Photonic Crystals
}

\section{Andriy E. Serebryannikov ( $\sim$ andser@amu.edu.pl )}

Adam Mickiewicz University

\section{Diana C. Skigin}

Universidad de Buenos Aires, Universidad de Buenos Aires

Guy A. E. Vandenbosch

Katholieke Universiteit Leuven

Ekmel Ozbay

National Institute of Materials Sciences and Nanotechnology (UNAM), Bilkent University

\section{Research Article}

Keywords: ESAT, deflection, specular reflection, photonic crystal (PhC), TE polarization

Posted Date: October 13th, 2021

DOl: https://doi.org/10.21203/rs.3.rs-948548/v1

License: (1) (1) This work is licensed under a Creative Commons Attribution 4.0 International License.

Read Full License 


\title{
Multifunctional Gratings for Multiband Spatial Filtering, Retroreflection, Splitting and Demultiplexing Based on $C_{2}$ Symmetric Photonic Crystals
}

\author{
Andriy E. Serebryannikov ${ }^{1, *}$, Diana C. Skigin ${ }^{2}$, Guy A. E. Vandenbosch ${ }^{3}$, and Ekmel \\ Ozbay $^{4}$
}

${ }^{1}$ Division of Physics of Nanostructures, ISQI, Faculty of Physics, Adam Mickiewicz University, 61-614 Poznan, Poland

${ }^{2}$ Universidad de Buenos Aires, Faculdad de Ciencias Exactas y Naturales, Departamento de Fisica, Grupo de Electromagnetismo Aplicado and CONICET-Universidad de Buenos Aires, Institute de Fisica de Buenos Aires (IFIBA), Buenos Aires, Argentina

${ }^{3}$ WaveCoRe research group, Electrical Engineering Department (ESAT), Katholieke Universiteit Leuven, 3001 Leuven, Belgium

${ }^{4}$ Nanotechnology Research Center (NANOTAM), National Institute of Materials Sciences and Nanotechnology (UNAM), Department of Physics, Department of Electrical Engineering, Bilkent University, 06800 Ankara, Turkey *andser@amu.edu.pl

\begin{abstract}
The concept of multifunctional reflection-mode gratings based on rod-type photonic crystals with $C_{2}$ symmetry is introduced and examined. The specific modal properties lead to the vanishing dependence of the first-negative-order maximum on the angle of incidence within a wide range, and the nearly sinusoidal redistribution of the incident-wave energy between zero order (specular reflection) and first negative diffraction order (deflection) at frequency variation that are the key features enabling various functionalities in one structure and functionality merging. The elementary functionalities offered by the studied structures, of which multifunctional scenarios can be designed, include but are not restricted to multiband spatial filtering, multiband splitting, and demultiplexing. The proposed structures are shown to be capable in multifunctional operation in case of an obliquely incident polychromatic wave. The generalized demultiplexing is demonstrated for the case when several polychromatic waves are incident at different angles. The same deflection properties yield multiband splitting, and merging demultiplexing and splitting functionalties in one functionality, which may contribute to various multifunctional scenarios. The proposed gratings are also studied in transmissive configuration.
\end{abstract}

\section{Introduction}

Finite-thickness slabs of a photonic crystal $(\mathrm{PhC})$ known as $\mathrm{PhC}$ gratings started attracting the interest more than one decade ago $^{1-4}$. PhC gratings and metamaterial based gratings with one-side corrugations have been well known, first of all, due to the structurally nonsymmetric designs enabling asymmetric transmission, which is connected with one-way deflection yielded by incident-wave energy redistribution in favor of a higher diffraction order(s $)^{5-7}$. Therefore, deflection can serve as the main enabler of diverse functionalities in PhC gratings. The common effect of dispersion of a Floquet-Bloch mode and diffractions at the corrugated interfaces of $\mathrm{PhC}$ gratings and metamaterial based gratings may lead to advanced functionalities. It is noteworthy that in the early-stage studies of the deflecting (blazed) gratings, the emphasis has been put on the operation at the Bragg condition $^{8-10}$. Later, this restriction has been mitigated in blazed gratings and $\mathrm{PhC}$ gratings ${ }^{11-14}$. Together with the novel blazed gratings ${ }^{12,13,15}$ and recently proposed metasurfaces ${ }^{16,17}$ and meta-gratings ${ }^{18}$ that are capable in deflection at rather arbitrary choice of geometric parameters and angle of incidence, PhC gratings suggest significant extension to the variety of approaches to wavefront manipulation.

Structures capable in multifunctional operation become trendy due to prospective solutions offered for device miniaturization and system integration ${ }^{18-20}$. Multifunctionality in $\mathrm{PhC}$ gratings assumes that different wave processes or groups of processes can be separated in space ${ }^{21}$ and/or in the frequency domain ${ }^{5}$. The recently proposed metasurfaces offer multifunctional scenarios that include but are not restricted to the ones with different functionalities or different manifestations of the same 
functionality in the neighboring frequency ranges ${ }^{22-24}$, at different incidence angles ${ }^{17,25}$, or at different polarization states ${ }^{26-28}$. Two functionalities can be merged into one at a fixed frequency ${ }^{29-32}$. The multifunctional scenarios that have been recently studied by numerous research groups include the elementary functionalities like focusing, deflection, polarization manipulation, splitting, and vortex and Bessel beam generation. Very recently, periodic meta-arrays with wideband and simultaneously wide-angle deflection that enables spatial filtering and wide-angle splitting and other functionalities in one structure have been demonstrated in reflection mode ${ }^{18}$. The earlier examples of PhC gratings with functionality integration include those yielding one-way splitting, spatial separation of two wave processes, and diodelike asymmetric transmission with opposite directions of strong transmission at two close frequencies ${ }^{5,21,30}$. Examples of similar integration have also been known for other configurations based on $\mathrm{PhCs}^{23,33,34}$. Although metasurfaces can suggest electrically thinner performances than $\mathrm{PhCs}$, it is still unclear whether they may replicate all functionalities achievable in PhCs or not. Owing to the diverse modal properties, PhCs may serve as a good platform for multifunctional devices.

In the present paper, the concept of $\mathrm{PhC}$ gratings backed with a metal reflector, which are capable of new multifunctional scenarios, is proposed and numerically validated. The goal of this study is demonstration of multifunctional scenarios achievable by using a specific Floquet-Bloch mode of the PhCs. The desired modal propertiescan be obtained in the PhCs with a rectangular lattice, i.e. with the $C_{2}$ symmetry, for which diffractions are achieved due to a larger lattice constant along the interface direction (i.e. $a_{x}>a_{y}$ ). The focus in this study will be put on the multifunctional scenarios that are expected to be unachievable in quasi-planar structures and the earlier proposed $\mathrm{PhC}$ gratings. It will be demonstrated that (i) the incidence-angle insensitive -1st-order deflection and (ii) the frequency selective -1st-order deflection together with the 0th-order specular reflection enable multiband bandpass and bandstop spatial filtering, partial or full demultiplexing, and multiband splitting in one structure, within the same frequency range. Noticeably, spatial filtering ${ }^{35-44}$, demultiplexing ${ }^{4-49}$ and splitting ${ }^{50-56}$ belong to the basic functionalities which are commonly demanded in photonics, optical communications, etc. while PhCs suggest a suitable platform for their realization. We will demonstrate how the above listed functionalities can be efficiently integrated in one structure. Moreover, multiband retroreflection and multiplexing can be obtained in the same structure, as well as the generalized demultiplexing and merging of demultiplexing and splitting functionalities into one functionality, by using the same physics and the same modal regime. The transmission properties of the same $\mathrm{PhC}$ grating but without a reflector will also be discussed. It should be noted that even if some of the elementary functionalities studied here can be obtained in structures of other types, low-symmetric PhCs are used here in order to enable all or most of the considered elementary functionalities at a given frequency range in one device, according to the purposes of this study. For instance, some of demultiplexing scenarios might be obtained by using volume Bragg gratings ${ }^{57,58}$. However, it can then be a challenge to obtain multiband spatial filtering, like that proposed in this paper, in the same structure and at the same frequency range. To calculate reflectance and transmittance, the integral equation technique with well justified and controllable accuracy and convergence has been used for the calculations ${ }^{59}$. Since the general dispersion properties of PhCs with $C_{2}$ symmetry are known, a study of dispersion is beyond the scope of this paper. It is organized as follows. The Results section that follows Introduction is divided by four subsections which present, consequently, proposed geometry, multiband spatial filtering and retroreflection, partial and generalized demultiplexing and multiband splitting, and $\mathrm{PhC}$ gratings without reflector. Then, the short Discussion and Methods sections are presented.

\section{Results}

\section{Proposed Geometry}

The general geometry of the proposed grating is shown in Fig. 1. It represents a finite-thickness slab of PhC that has $C_{2}$ symmetry, which is placed above the metal reflector. Structures with such a symmetry are known for self-collimation and other fascinating propagation effects ${ }^{50,60-64}$. In this context, the canalization of electromagnetic waves should also be mentioned ${ }^{65}$. The studied structure is assumed to be periodic and infinitely long in the $x$ direction. It is illuminated from the upper half-space by a plane wave incident obliquely at angle $\theta$. Consideration is restricted to TE polarization, i.e. electric field vector is along the $z$ axis. The rods are assumed to be made of a dielectric material with relative permittivity $\varepsilon_{r}$. Besides, it is assumed that a wide variety of the natural dielectric materials can be used to fabricate the PhC.

The $x$ and $y$ components of the PhC lattice vector components are denoted by $a_{x}$ and $a_{y}$, respectively; here $a_{x}=2 a_{y}$. Thus, the grating period $L$ is equal to $a_{x}$. The ratio $d / a_{y}=0.4$ where $d$ is rod diameter is used throughout the paper. For instance, at $L=a_{x}=1.5 \mu \mathrm{m}$, we have $a_{y}=750 \mathrm{~nm}$ and $d=300 \mathrm{~nm}$. The other values of $d / a_{y}$ have also been examined. The host medium is assumed to be air. The only requirement to the back-side reflector is that it should be weakly absorptive and weakly transmissive that is achieved for a very wide variety of materials and geometrical parameters. For the sake of definiteness, it is assumed here that the reflector is made of a Drude metal with permittivity $\varepsilon_{m}=1-\omega_{p} /[\omega(\omega-i \gamma)]$, where $\omega_{p}$ is plasma frequency and $\gamma$ is collision frequency; $\omega_{p} a_{y} / c=20 \pi$ and $\gamma / \omega_{p}=0.01$. 


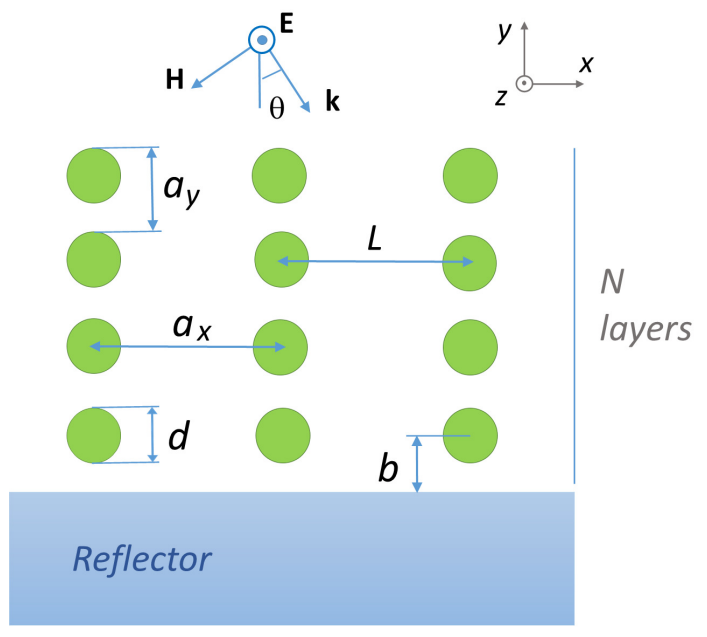

Figure 1. General geometry of PhC grating with back-side reflector, $a_{x}=2 a_{y}=L$. Three periods over $x$ are shown.
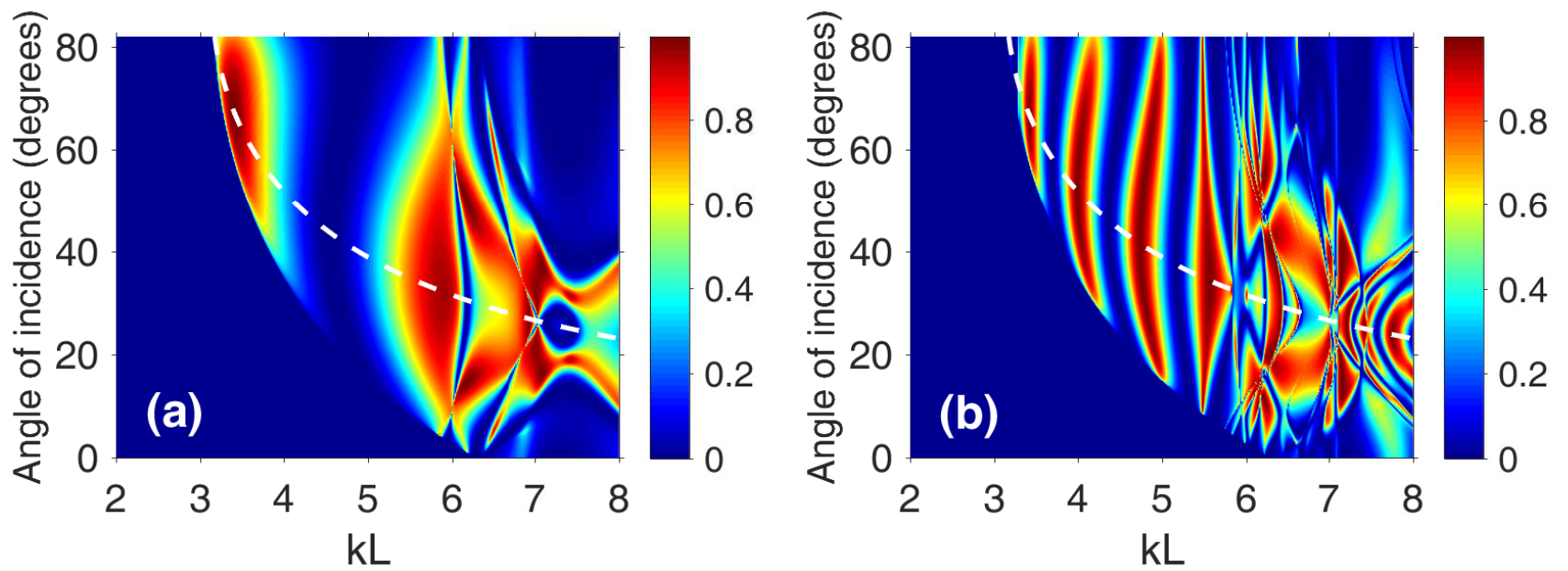

Figure 2. First-negative-order reflectance, $r_{-1}$, at the simultaneous variation of $k L$ and $\theta$ for the PhC grating with (a) $N=2$, (b) $N=6, \varepsilon_{r}=11.4$; the grating is backed with a reflector. Dashed white lines correspond to retroreflection regime, in which $\phi_{-1}=-\theta$.

\section{Multiband Spatial Filtering and Retroreflection}

In this section, we demonstrate the potential of the proposed structures that are based on $C_{2}$ symmetric PhCs in multiband spatial filtering and retroreflection. These functionalities belong to the elementary functionalities, which serve as the building blocks for multifunctional scenarios. Since far-field operation is considered, we use zero-order and higher-order reflectances to quantify the wave reflection, see Methods. For plane-wave illumination, spatial filtering can be considered as an analogue of frequency-domain filtering, but in the $\theta$-domain, assuming that $f=$ const, see Ref. 35. Various mechanisms and structures have been proposed for reflection and transmission modes that enable low-pass, bandpass, and bandstop spatial filtering. At beam illumination, the contribution of the individual angular components is modified, so that the beam reshaping takes place, e.g. see Ref. 66. In reflection mode, rather simple but carefully designed gratings have been suggested to obtain wideband and simultaneously wide-angle plane-wave deflection by using the reflected order $m=-1$ to yield wideband spatial filtering ${ }^{15,18}$. Here, the goal is different. Instead of obtaining a single and large area of $r_{-1} \approx 1$ in $(k L, \theta)$-plane, it is obtaining multiple regions of $r_{-1} \approx 1$, which are intermittent with the regions of $r_{0} \approx 1$ (i.e. small $r_{-1}$ ).

The results for the first-negative-order reflectance, $r_{-1}$, vs. $k L(k=\omega / c$ is the free-space wavenumber, $\omega=2 \pi f$ is the angular frequency, $f$ is frequency, $c$ is the speed of light), are presented in Fig. 2. The "mountains" of $r_{-1} \approx 1$ and "valleys" of small $r_{-1}$ are the main features observed here. The "valleys" of small $r_{-1}$ correspond to the "mountains" of $r_{0}$ (not shown), since transmission is negligible. From the spatial filtering perspective, it is important that we have non-bent "mountains", i.e. 

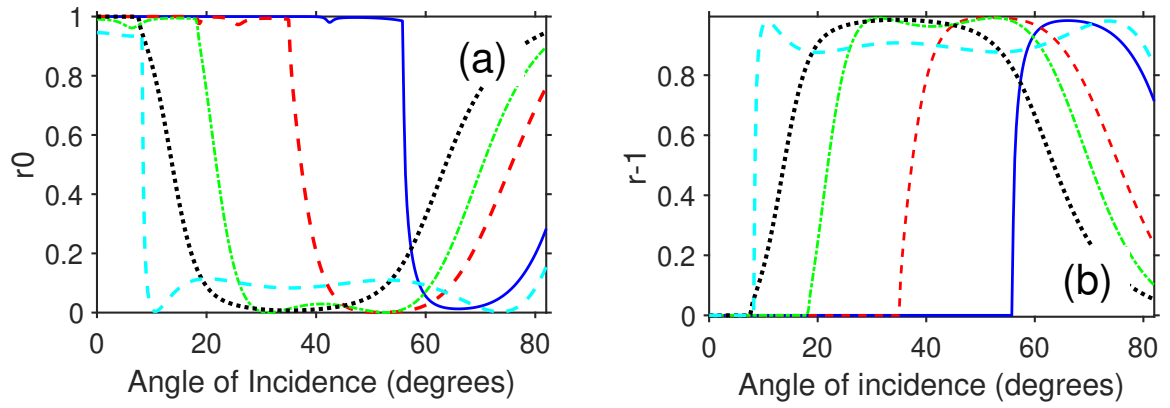

Figure 3. (a) Zero-order reflectance, $r_{0}$, and (b) first-negative-order reflectance, $r_{-1}$ as a function of $\theta$ for the same structure as in Fig. 2. Solid blue, dashed red, dash-dotted green, dashed cyan lines, and dotted black lines correspond to $k L=3.434$, $3.99,4.783,5.486$, and 5.546, respectively.

spectral location of the maxima of $r_{-1}$ should not be changed while varying $\theta$ in a wide range. This behavior corresponds to the flat dispersion occurring within a limited range of wavenumber variation. It indicates, among others, the possibility of a nearly sinusoidal energy exchange between $r_{0}$ and $r_{-1}$ when $f$ is varied, and that the exchange scenario can weakly depend on $\theta$.

The regimes of $r_{-1} \approx 1$ enable multiband bandpass filtering in the $\theta$-domain. The complementary regions of $r_{0} \approx 1$ that occur in the same spectral regimes but for smaller $\theta$ yield bandstop filtering. The effect of $N$ manifests itself in the different numbers of the intermittent regions of $r_{0} \approx 1$ and $r_{-1} \approx 1$. There are two regions of large $r_{-1}$ near $k L=3.4$ and 5.9 in Fig. 2(a), and four such regions around $k L=3.42,4.0,4.75$, and 5.55 in Fig. 2(b). Interestingly, similar features have been observed for transmission-mode spatial filtering in diffraction-free PhCs, where the intermittence occurs for $t_{0}$ and $r_{0}{ }^{35}$. In our case, the reflected order $r_{-1}$ plays the same role in spatial filtering as $t_{0}$ does in Ref. 35.

Note that $\phi_{-1}$ may vary in a very wide range for each "mountain". For instance, at $k L=5.486$, we obtain $\phi_{-1}=-81.4^{\circ}$ at $\theta=9^{\circ}$ and $\phi_{-1}=-8.9^{\circ}$ at $\theta=82^{\circ}$, in agreement with the Lorentz reciprocity. At $k L=4.05$, we obtain $\phi_{-1}=-71^{\circ}$ at $\theta=37^{\circ}$ and $\phi_{-1}=-38.2^{\circ}$ at $\theta=68^{\circ}$. Such a wide range of the output-wave angle makes possible multiband retroreflection regime that is achieved when $\phi_{-1}=-\theta$ and $\sin \phi_{-1}=-\pi / k L^{67-69}$. This regime is known as Littrow mounting. As observed in Fig. 2, retroreflection occurs within each "mountain", so that the number of the retroreflection bands depends on $N$. In particular, retroreflection is observed at $\theta=51^{\circ}$ when $k L=4.05$, and at $\theta=34.9^{\circ}$ when $k L=5.486$. Moreover, the neighboring $k L$-ranges, e.g. $6<k L<8$, which corresponds to another Floquet-Bloch mode, may also contribute to the resulting multibandness. Interestingly, $r_{-1}>0.9$ for $\phi_{-1}=-\theta$ also when the order $m=-2$ may propagate, according to Eq. (6). The multiband retroreflection in Fig. 8 differs from wide-band retroreflection in Ref. 18, since here it may occur only at discrete pairs of $k L$ and $\theta$ values.

For more evidence, Fig. 3 presents $r_{0}$ and $r_{-1}$ at the selected values of $k L$, which correspond to the tops of the "mountains" in Fig. 2(b). As observed, we obtain bandstop spatial filtering for $r_{0}$ and bandpass spatial filtering for $r_{-1}$. It is seen that $r_{-1}>0.9$ in a wide $\theta$-range. The boundaries of the $\theta$-domain bands are moderately sharp, and the the upper- $\theta$ boundary is more blurred than the lower one. The width and location of the $\theta$-domain band depends on the choice of the "mountain". The location of the lower- $\theta$ boundary in Fig. 3(b) can vary from $\theta=10^{\circ}$ to $60^{\circ}$. One can see that the band widening is possible at the price of a decrease of $\max r_{-1}$ and a less smooth shape of $r_{-1}$ vs. $\theta$, e.g., compare to the cases of $k L=5.486$ and $k L=5.546$ in Fig. 3(b). This remains true for all four "mountains". The features observed in Figs. 2 and 3 can be obtained for a wide variety of the $\mathrm{PhC}$ rod materials, e.g. for $5.6<\varepsilon_{r}<12.5$. As shown in the next section, partial and generalized demultiplexing and multiband splitting can be obtained in the same structure and at the same frequency range as multiband spatial filtering and retroreflection, i.e. without additional parameter adjustment, that makes the proposed $\mathrm{PhC}$ gratings highly capable in multifunctional operation.

\section{Partial and Generalized Demultiplexing and Multiband Splitting}

In this section, we demonstrate the potential of the same and similar structures in demultiplexing and multiband splitting, which represent the second part of the selected elementary functionalities. Figure 4 presents zero-order and first-negative-order reflectance, $r_{0}$ and $r_{-1}$, vs. normalized frequency $k L$ for a PhC grating created by a finite-thickness slab of PhC with $C_{2}$ symmetry, which is backed by a metal reflector. Structures with different numbers of the rod layers, $N$, were compared. As expected, the nearly sinusoidal energy exchange between the orders $m=0$ and $m=-1$ occurs at varying frequency. Notably, this feature is rather uncommon, because it needs the specific modal properties. For the energy exchange of such a kind, the maxima of $r_{-1} \approx 1$, at which the almost total conversion of the incident-wave energy into a deflected beam outgoing at the angle $\phi_{m}\left(m=-1, \phi_{-1} \neq \theta\right)$ takes place, are intermittent with the specular reflection regimes of $r_{0} \approx 1\left(\phi_{0}=-\theta\right)$, when 

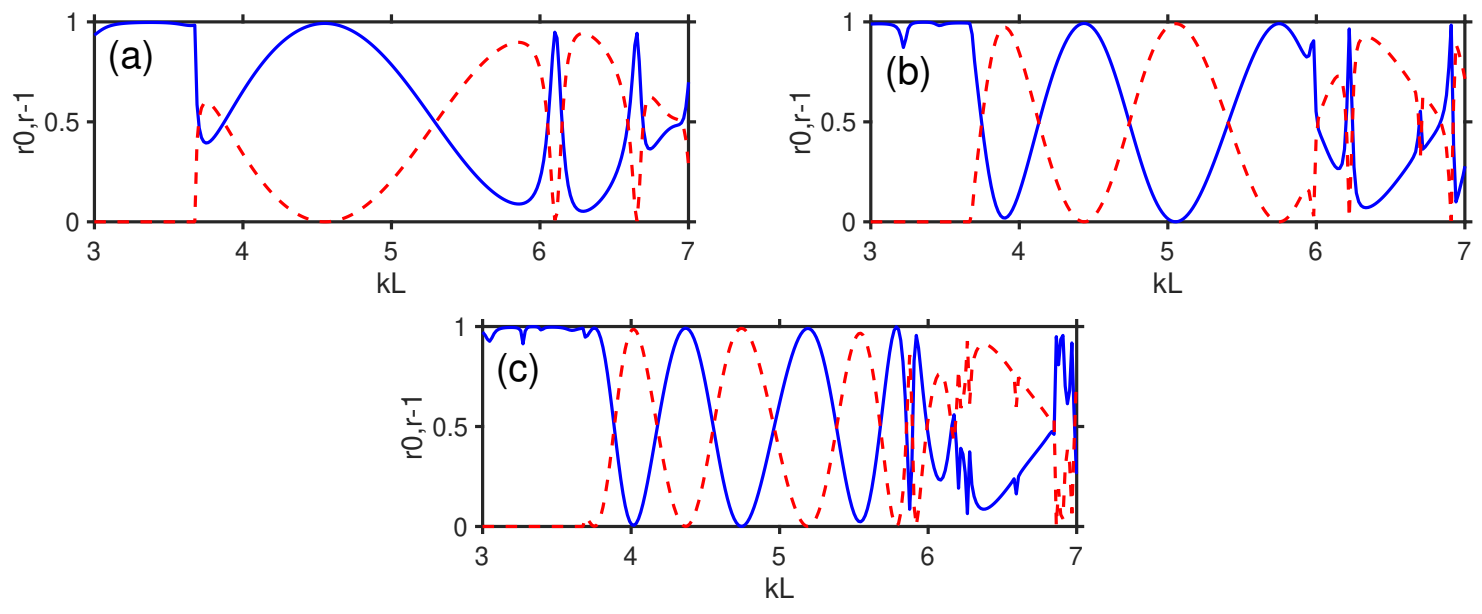

Figure 4. Reflectance for PhC grating with (a) $N=2$, (b) $N=4$, (c) $N=6, \varepsilon_{r}=11.4, \theta=45^{\circ}$; the grating is backed with a reflector; solid blue line - $r_{0}$, dashed red line $-r_{-1}$.

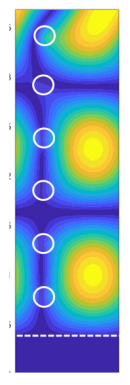

(a)

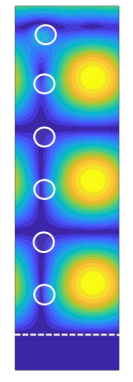

(b)

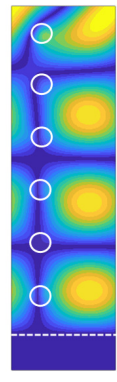

(c)

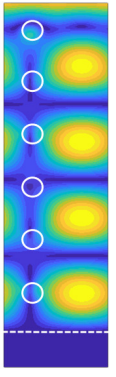

(d)

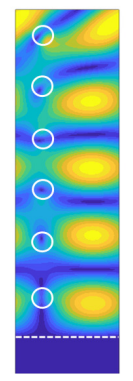

(e)

Figure 5. Examples of electric field distribution within a one period over $x$, at $N=6, \varepsilon_{r}=11.4, \theta=45^{\circ}$; (a) $k L=4.01$, (b) 4.37 , (c) 4.75 , (d) 5.19, and (e) 5.54. Local field maxima are observed at the mid-distance between the neighboring rods in $x$ direction. The dashed white line indicates the upper surface of the metal reflector.

$3.8<k L<5.7$, in agreement with Fig. 2. The number of the reflection regimes of each of the two types depends on $N$. For $N=2$, we have one reflection region with $r_{0} \approx 1$ and one with $r_{-1} \approx 1$. For $N=4$, there are two regimes of $r_{-1} \approx 1$ and two regimes of $r_{0} \approx 1$ in the same $k L$-range. For $N=6$, there are three regimes of $r_{-1} \approx 1$ and three regimes of $r_{0} \approx 1$.

The observed intermittence reminds us about Fabry-Perot interferences in a finite-thickness slab of a homogeneous dielectric material, but here we have $r_{0}$ and $r_{-1}$ instead of $r_{0}$ and $t_{0}$. Figure 5 presents electric field distribution within one period of the structure along the $x$ axis. The values of $k L$ are chosen that correspond to the maxima of $r_{0} \approx 1$ and $r_{-1} \approx 1$ in Fig. 4 (c). Figures 5(a), 5(c), and 5(e) correspond to $r_{-1} \approx 1$, Figs. 5(b) and 5(d) correspond to $r_{0} \approx 1$. The observed features give evidence of the interferential nature of the mechanism of energy exchange between the orders $m=0$ and $m=-1$. Here, the equivalent grating thickness $D \approx l \lambda_{\mathrm{PhC}} / 2$ is needed to obtain $r_{0} \approx 1$, while $D \approx \lambda_{\mathrm{PhC}} / 4+l \lambda_{\mathrm{PhC}} / 2$ leads to $r_{-1} \approx 1\left(\lambda_{\mathrm{PhC}}\right.$ is the wavelength in $\mathrm{PhC}$ along the $y$ direction, $l$ is integer). Thus, these two conditions correspond to the constructive and destructive interferences in a non-backed homogeneous dielectric slab where they lead to $t_{0}=1$ and a maximum of $r_{0}$. Note that the various effects of interferences have been studied in PhCs of different types ${ }^{5,70,71}$. On the other hand, the observed features may remind a Gires-Tornouis interferometer. However, in contrast with its standard version, we utilize here the orders $m=0$ and $m=-1$ together with the specific modal properties enabling the desired multifunctional scenarios.

The observed behavior of $r_{0}$ and $r_{-1}$ in Fig. 4 and that of $r_{-1}$ in Fig. 2 can be used for partial demultiplexing, as illustrated by the schematic in Fig. 6. Let the wave incident at a given angle $\theta$ represents a sum of several spectral components, which correspond to (nearly) perfect deflection, $r_{-1} \approx 1$ (frequencies $f_{1}^{d}, f_{2}^{d}, \ldots f_{P}^{d}$ ), and several spectral components corresponding 


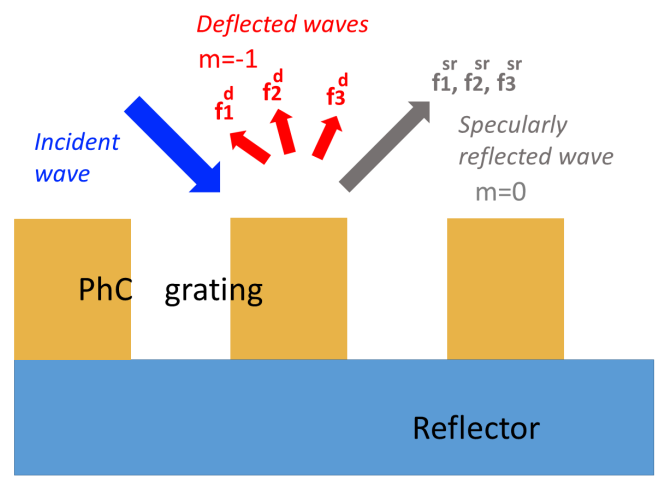

Figure 6. Schematic illustrating the merging of partial demultiplexing and splitting functionalities at given $\theta$. Weak dependence of the maxima of $r_{-1}$ on $\theta$ makes possible the use of several polychromatic waves incident at different angles, for each of which the merging of partial demultiplexing and splitting functionalities can be obtained.
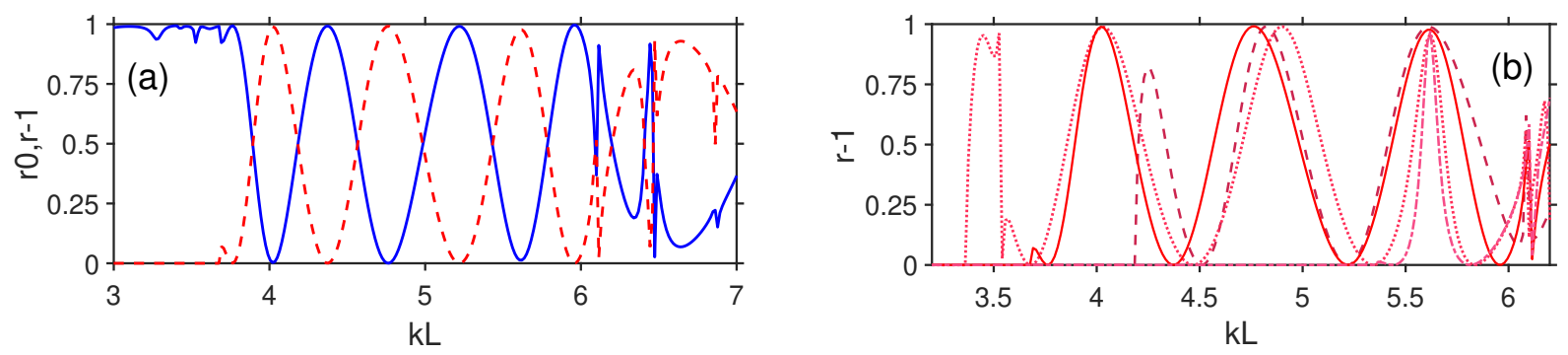

Figure 7. Reflectance for the PhC grating $\varepsilon_{r}=9.6$ and $N=6$; (a) solid blue line $-r_{0}$, dashed red line $-r_{-1} ; \theta=45^{\circ}$; (b) $r_{-1}$; solid line - $\theta=45^{\circ}$, dashed line - $\theta=30^{\circ}$, dotted line - $\theta=60^{\circ}$, dash-dotted line - $\theta=10^{\circ}$; reflector is placed on the back side.

to the specular reflection regime, $r_{0} \approx 1$ (frequencies $f_{1}^{s r}, f_{2}^{s r}, \ldots f_{Q}^{s r}$ ), i.e. the incident wave is presented as

$$
u(t, \mathbf{r})=\sum_{p=1}^{P} A_{p} \exp \left(-i 2 \pi f_{p}{ }^{d} t+i \mathbf{k r}\right)+\sum_{q=1}^{Q} A_{q} \exp \left(-i 2 \pi f_{q}^{s r} t+i \mathbf{k r}\right)
$$

where $A_{p}$ and $A_{q}$ are the amplitudes, $\mathbf{k}$ is the wave vector, and $\mathbf{r}$ is the generalized coordinate.

Then, the components with $f=f_{p}^{d}$ will be deflected at the $k L$-dependent angles $\phi_{m}$ given by Eq. (6) at $m=-1$. In turn, all of the components with $f=f_{q}^{s r}$ will be specularly reflected, so that a new, reduced sum of spectral components will be formed. In such a way, the first part of the incident wave is demultiplexed, while the second part is not. Clearly, the demultiplexed (deflected) and nondemultiplexed (specularly reflected) parts are forwarded to the different quadrants of the upper half-space. Thus, the discussed functionality can also be understood in the context of sorting, i.e. separation of the spectral components of the incident wave by redirecting them to different sectors. For instance, for the regimes of $r_{-1} \approx 1$ in Fig. 4(c), we obtain $\phi_{-1} \approx-59^{\circ},-38^{\circ}$, and $-25^{\circ}$, respectively, at $k L=4.01,4.75$, and 5.54 , while $\phi_{-1} \approx 45^{\circ}$ for the regimes of $r_{0} \approx 1$. The incident wave can only be a sum of the spectral components with $f=f_{i}^{d}$, or a sum the specular-reflection components with $f=f_{i}^{s r}$, see Eq. (6). Then, in the former case, we obtain a full demultiplexing, i.e. all spectral components correspond to the different values of $\phi_{-1}$. In the latter case, we have conventional specular reflection. Accordingly, the retroreflection regime is excluded from the set of the outgoing waves.

The same structures suggest multiband splitting when $r_{0}=r_{-1} \approx 0.5$. If the incident wave represents a sum of the spectral components, for each of which splitting may occur (frequencies $f_{1}^{s p}, f_{2}^{s p}, \ldots f_{S}^{s p}$ ), i.e.

$$
u^{s p}(t, \mathbf{r})=\sum_{s=1}^{S} A_{s} \exp \left(-i 2 \pi f_{s}^{s p} t+i \mathbf{k r}\right),
$$

where $A_{s}$ is amplitude, then the splitting and demultiplexing functionalities can be merged in one step. In other words, half of the incident-wave energy is converted into specular reflection, and the other half is spatially demultiplexed. Each of the halfs 

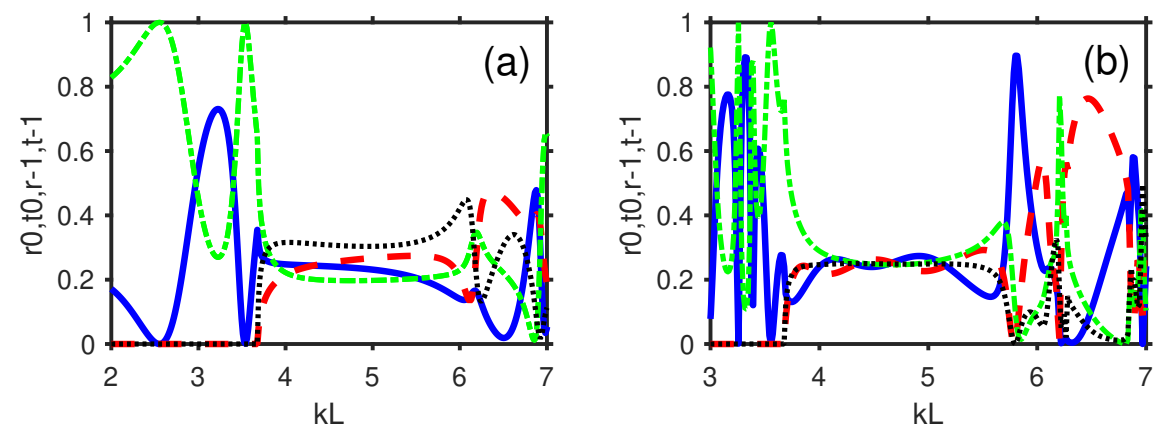

Figure 8. Zero-order reflectance, $r_{0}$ (blue solid line), first-negative-order reflectance, $r_{-1}$ (red dashed line), zero-order transmittance, $t_{0}$ (green dash-dotted line), and first-negative-order transmittance, $t_{-1}$ (black dotted line) vs. $k L$, at (a) $N=2$ and (b) $N=6, \varepsilon_{r}=11.4 ; \theta=45^{\circ}$; there is no back-side reflector.

comprises the same spectral components as in Eq. (6) but with the halved amplitudes. In Fig. 4(c), it happens for $\phi_{-1} \approx-65^{\circ}$, $-53^{\circ},-42^{\circ},-34^{\circ},-27^{\circ},-23.5^{\circ}$, and $-21.5^{\circ}$, respectively, at $k L=3.89,4.18,4.55,4.97,5.39,5.68$, and 5.85. Also here no one of the regimes of $r_{-1} \approx 1$ corresponds to the Bragg condition. Moreover, the input signal may represent a sum of the components with $f_{q}^{s r}, f_{p}^{d}$, and $f_{s}^{s p}$, leading to even more complex multifunctionalty. It is noteworthy that the full demultiplexing can also be obtained in reflective quasi-planar meta-arrays like the ones in Ref. 15, 18, but rather for a single wide band of large $r_{-1}$, so that some of the selected elementary functionalities, i.e. partial demultiplexing and multiband splitting are not achievable therein.

Partial demultiplexing and multiband splitting can be tuned in the simplest way (i.e. without tunable materials) by varying $\theta$. Since both $\phi_{-1}$ and the $k L$ threshold for $\left|\sin \phi_{-1}\right| \leq 1$ depend on $\theta$ [see Eq. (6)], the width of the $\phi_{-1}$ range that is achievable in the used energy exchange regime can be varied with $\theta$. The weak dependence of $k L$-values corresponding to the maxima on $\theta$, like in Fig. 2(b), makes possible generalization of demultiplexing, for which several polychromatic waves (instead of one polychromatic wave like that shown in Fig. 6) are incident at different angles, each comprising multiple spectral components [like in Eq. (1) for one wave]. Such generalization may be difficult when using structures of other types, e.g., volume Bragg gratings, while the desired modal properties can be offered, for instance, by $C_{2}$ symmetric PhCs. The possibility of demultiplexing in the framework this scenario is demonstrated in Fig. 7 for the PhC grating with $\varepsilon_{r}=9.6$. Figure 7(a) presents $r_{0}$ and $r_{-1}$ vs. $k L$ at $\theta=45^{\circ}$. The results are similar to those in Fig. 4, in accordance with the fact that the observed energy exchange between the orders $m=0$ and $m=-1$ takes place in a wide range of $\varepsilon_{r}$ variation, e.g., from 5.6 to 12.5. Next, Fig. 7(b) shows $r_{-1}$ vs. $k L$ for four selected values of $\theta$. The feature common for all values of $\theta$ is the intermittence of the maxima of $r_{-1} \approx 1$ and $r_{0} \approx 1$, see Fig. 2(b) for comparison. The maxima of $r_{-1}$ of two types can be distinguished in Fig. 7(b). For the first one, spectral locations are the same for the maxima that correspond to different $\theta$; for the second one, spectral locations are slightly different. Nevertheless, they both may contribute to the demultiplexing scenarios, in which several polychromatic waves are incident at different angles. For the case shown in Fig. 7(b), we obtain $\phi_{-1}=-58.9^{\circ}$ and $-44.2^{\circ}$, respectively, at $\theta=45^{\circ}$ and $60^{\circ}$ for $k L=4.02 ; \phi_{-1}=-52.7^{\circ},-36^{\circ}$ and $-25.4^{\circ}$ at $\theta=30^{\circ}, 45^{\circ}$ and $60^{\circ}$ for $k L=4.85$; and $\phi_{-1}=-70.8^{\circ},-38.2^{\circ},-24.3^{\circ}$ and $-14.6^{\circ}$ at $\theta=10^{\circ}, 30^{\circ}, 45^{\circ}$ and $60^{\circ}$ for $k L=5.62$. By adjusting the structural parameters and choosing proper values of $\theta$, a difference in the values of $\phi_{-1}$ that exceeds a desired threshold value can be obtained. On the other hand, an adjustment may lead to the case when two or more waves incident with different pairs of $(k L, \theta)$ are deflected to the same direction, so that multiplexing/beam combining can be obtained. Clearly, not only conventional but also generalized demultiplexing can be a part of the complex multifunctional scenarios which involve spatial filtering, retroreflection, and/or splitting.

\section{PhC grating without reflector}

In this section, we briefly discuss the transmission properties of the designed $\mathrm{PhC}$ gratings. The structure studied here is easily obtained from the basic geometry in Fig. 1 by removing the back-side reflector. Figure 8 presents the results for $r_{0}, r_{-1}, t_{0}$, and $t_{-1}$ vs. $k L$ for the PhC grating with the same parameters as in Figs. 4(c), 2(b), 3. The most interesting feature is that all four diffraction efficiencies vary weakly in the $k L$-range, in which we observed the energy exchange between -1 st and 0 th orders in case with the reflector. Moreover, their values are close to each other for $N=6$, say, at $4.2<k L<5.2\left(-52^{\circ}<\phi_{-1}<-30^{\circ}\right)$, and that enables a four-beam wideband splitter.

Finally, Fig. 9 presents the maps of $t_{-1}$ and $r_{-1}$ in $(k L, \theta)$-plane. As observed, the features initially found in Fig. 8 remain the same within the large region, although $t_{-1}$ varies weaker than $r_{-1}$. Thus, the nearly equal efficiencies are not a result of an 

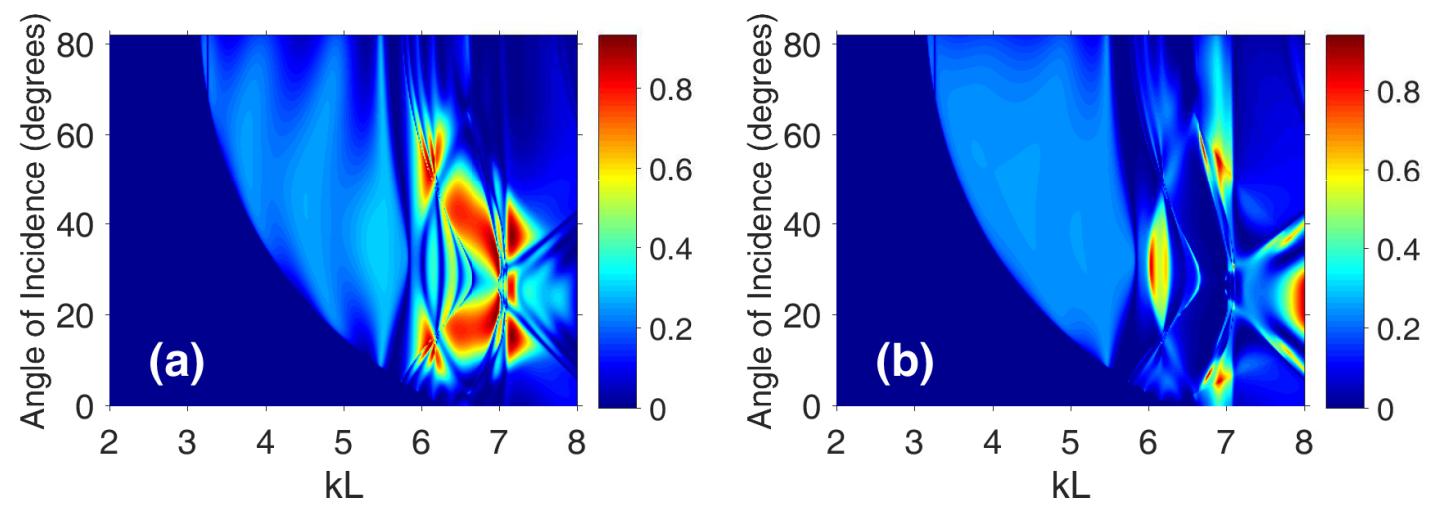

Figure 9. (a) First-negative-order reflectance, $r_{-1}$, and (b) first-negative-order transmittance, $t_{-1}$, at simultaneous variation of $k L$ and $\theta ; N=6, \varepsilon_{r}=11.4$; there is no back-side reflector.

accidental choice of $\theta$ and $k L$. Rather, it reflects the nature of the used modal regime. It is noteworthy that a similar behavior has been observed in a wide range of $\varepsilon_{r}$ variation (results are not shown). Interstingly, the behavior of $r_{0}, r_{-1}, t_{0}$, and $t_{-1}$ outside the range $3.8<k L<5.7$ is completely different. It results from the difference in the properties of Floquet-Bloch modes, whose analysis is beyond the scope and can be a subject of a future study.

\section{Discussion}

We studied the PhC gratings backed with a reflector that are capable of multifunctional operation, so that several functionalities can be achieved in one structure, in the same frequency range. The specific modal properties that result from the $C_{2}$ symmetry of the used PhCs yield co-existense of angle-independent deflection and specific energy exchange between zero and first negative orders while varying frequency that serve as the main enablers of the multifunctional scenarios. Thus, there may be no full analogue of the proposed combination of functionalities in the earlier works. An important advantage is that the two feature mentioned above are kept within a large region in $(k L, \theta)$-plane. In particular, this allows to select proper widths of the passbands in the $\theta$-domain and achieve quite a large angular difference between the individual demultiplexed (outgoing) waves or input waves (in case of multiplexing). The most interesting manifestation is probably the nearly perfect, multiband, wide-angle deflection in the first negative diffraction order regime that enables multiband bandpass filtering in the $\theta$-domain. Up to three spectral regimes of the nearly ideal deflection that are robust to the $\theta$ variations and intermittent with the spectral regimes of specular reflection have been numerically demonstrated, while the energy exchange between the propagating zero and first negative orders in reflection is similar to that between reflectance and transmittance in case of the interferences in a homogeneous transmissive dielectric slab. A larger number of the bands within the given frequency and/or incidence-angle ranges can be obtained by an increase of the rod layers. A polychromatic incident wave can experience either full or partial demultiplexing or specular reflection, depending on which spectral components are comprised. The generalization of demultiplexing for the case of several polychromatic waves incident at different angles is proposed that can also contribute to multifunctional scenarios. A multiband splitter is one more functionality enabled by first-negative-order deflection in the same frequency range. Moreover, demultiplexing and splitting functionalities can be merged in one functionality. The same structure can function as a multiplexer or a multiband retroreflector. The proposed structures capable in multifunctional operation can be used just for a single functionality, if required. The same structure but without the back-side reflector can show nearly equal efficiencies for all four propagating orders (zero and first-negative orders in transmission and reflection). The use of the both orthogonal polarizations can further reinforce the multifunctionality potential. The studied $\mathrm{PhC}$ gratings can be appropriate for fabrication, since fabrication techniques for two-dimensional $\mathrm{PhCs}$ are well developed ${ }^{73,74}$. The concept transfer to the $\mathrm{PhC}$ slabs will be considered at the next steps of this research program. The obtained results are expected to be important, first of all, from the multifunctionality perspective. They may be useful for the development of new infrared devices with a high degree of miniaturization and integration, and indicate a route to new applications for low-symmetric $\mathrm{PhCs}$ and $\mathrm{PhC}$ gratings.

\section{Methods}

The studied structure is assumed to be illuminated by a plane incident wave that is given by

$$
E^{i}(x, y)=E_{0} \exp \left(i \alpha_{0} x-i \beta_{0} y\right)
$$


where $E_{0}$ is the amplitude, $\alpha_{0}=k \sin \theta$, and $\beta_{0}=k \cos \theta$.

The electric field above the $\mathrm{PhC}$ grating is presented as follows:

$$
E^{+}(x, y)=E^{i}(x, y)+\sum_{m=-\infty}^{\infty} \rho_{m} \exp \left(i \alpha_{m} x+i \beta_{m} y\right),
$$

where $\beta_{m}=\sqrt{k^{2}-\alpha_{m}^{2}}, \operatorname{Im} \beta_{m} \leq 0, \alpha_{m}=\alpha_{0}+2 \pi m / L, \rho_{m}$ is the amplitude of $m$ th-order reflected wave. The transmitted electric field below the reflector is given by

$$
E^{-}(x, y)=\sum_{m=-\infty}^{\infty} \tau_{m} \exp \left(i \alpha_{m} x-i \beta_{m} y\right)
$$

where $\tau_{m}$ is the amplitude of the $m$ th-order transmitted wave. For a reflective configuration, $E^{-}$may be assumed to be zero at the appropriate choice of the material and the thickness of the reflector, but it is nonzero in the general case, i.e. when the reflector is semi-transparent or removed.

An integral equation technique is used for the purposes of numerical study. The problem is formulated and solved in the spectral domain with respect to the unknown amplitudes of spectral harmonics of the electric field within one structural period over $x$. Once the amplitudes at the upper and lower boundaries of the calculation region are found, the values of $\rho_{m}$ and $\tau_{m}$, including those at $m=0$ and $m=-1$, are directly calculated. Details of the problem formulation and iterative numerical procedure can be found in Ref. 59. Throughout the present paper, we use $m$ th-order reflectances, $r_{m}=\rho_{m} \rho_{m}^{*} \operatorname{Re} \beta_{m} / W$, and $m$ th-order transmittances, $t_{m}=\tau_{m} \tau_{m}^{*} \operatorname{Re} \beta_{m} / W$, in order to quantify far-field behavior. Here, $W$ is the energy of the incident wave and asterisk means complex conjugate. In the ideal (i.e., lossless) case, $R+T=W$ where $R=\sum_{m=-\infty}^{\infty} r_{m}$ is the overall reflectance and $T=\sum_{m=-\infty}^{\infty} t_{m}$ is the overall transmittance. In the reflective configuration, $T \approx 0$.

The angle of the $m$ th-order outgoing wave can be found from the grating equation ${ }^{72}$ :

$$
\sin \phi_{m}=\sin \theta+2 \pi m / k L
$$

where $m=0, \pm 1, \pm 2, \ldots$. Therefore, the structure can be designed so that a desired number of the diffraction orders formally may propagate, while the remaining ones are evanescent. In our $\mathrm{PhC}$ gratings, the orders $m=0$ and $m=-1$ may propagate, whereas the ones with $m>0$ and $m<-1$ remain evanescent. In this case, $R=\sum_{m=-1}^{0} r_{m}$ and $T=\sum_{m=-1}^{0} t_{m}$.

\section{Acknowledgements}

This work was supported by Narodowe Centum Nauki, Project UMO-2015/17/B/ST3/00118 and TUBITAK (program 2221). E.O. acknowledges partial support from the Turkish Academy of Sciences. A.E.S. would like to thank Dr. T. Magath and Dr. A. Yu. Petrov for the fruitful discussions.

\section{References}

1. Maystre, D. Photonic crystal diffraction gratings. Opt. Express 8, 209-216 (2001).

2. Collardey, S., Tarot, A.-C., Pouliguen, P. and Mahdjoubi, K. Use of electromagnetic band-gap materials for RCS reduction. Microwave Opt. Technol. Lett. 44, 546-550 (2005).

3. Serebryannikov, A. E., Magath, T. and Schuenemann, K. Bragg transmittance of $s$-polarized waves through finite-thickness photonic crystals with a periodically corrugated interface. Phys. Rev. E 74, 066607 (2006).

4. Mandatori, A., Bertolotti, M. and Sibilia, C. Asymmetric transmission of some two-dimensional photonic crystals. J. Opt. Soc. Am. B 24, 685-690 (2007).

5. Serebryannikov, A. E., Alici, K. B., Magath, T., Cakmak, A. O. and Ozbay, E. Asymmetric Fabry-Perot-type transmission in photonic-crystal gratings with one-sided corrugations at a two-way coupling. Phys. Rev. A 86, 053835 (2012).

6. Serebryannikov, A. E., Colak, E., Magath, T. and Ozbay, E. Two types of single-beam deflection and asymmetric transmission in photonic structures without interface corrugations. J. Opt. Soc. Am. A 33, 2450-2458 (2016).

7. Rodriguez-Ulibarri, P., Beruete, M., Navarro-Cia, M. and Serebryannikov, A. E. Wideband unidirectional transmission with tunable sign-switchable refraction and deflection in nonsymmetric structures. Phys. Rev. B 88, 165137 (2013).

8. Hessel, A., Schmoys, J., and Tseng, D. Bragg-angle blazing of diffraction gratings. J. Opt. Soc. Am. 65, 380-384 (1975).

9. Heath, J. W. and Jull, E. V. Perfectly blazed reflection gratings with rectangular grooves. J. Opt. Soc. Am. 68, 1211-1217 (1978). 
10. Jull, E. V., Heath, J. W. and Ebbeson, G. R. Gratings that diffract all incident energy. J. Opt. Soc. Am. 67, 557-559 (1977).

11. Chen, W., Beaulieu, N. C., Michelson, D. G. and Jull, E. V. Off-Bragg Blazed Rectangular Groove Gratings for High Diffraction Efficiency Devices. IEEE Trans. Ant. Propag. 61, 2342-2347 (2013).

12. Memarian, M., Li, X., Morimoto, Y. and Itoh, T. Wide-band/angle Blazed Surfaces using Multiple Coupled Blazing Resonances. Sci. Rep. 7, 42286 (2017).

13. Ribot, C., Lee, M. S. L., Collin, S., et al. Broadband and efficient diffraction. Adv. Opt. Mater. 1, 489-493 (2013).

14. Hemmatyar, O., Abbassi, M. A., Rahmani, B. and Memarian, M. Wide-Band/Angle Blazed Dual-Mode Metallic Groove Gratings. IEEE Trans. Ant. Propag. 69, 379-386 (2021).

15. Serebryannikov, A. E., Lalanne, Ph., Petrov, A. Y. and Ozbay, E. Wide-angle reflection-mode spatial filtering and splitting with photonic crystal gratings and single-layer rod gratings. Opt. Lett. 39, 6193-6196 (2014).

16. Jung, J., Park, H., Park, J., Chang, T. and Shin, J. Broadband metamaterials and metasurfaces: a review from the perspectives of materials and devices. Nanophoton. 9, 3165-3196 (2020).

17. Cheng, J. R., Inampudi, S. and Mosallaei, H. Optimization-based Dielectric Metasurfaces for Angle-Selective Multifunctional Beam Deflection. Sci. Rep. 7, 12228 (2017).

18. Aalizadeh, M., Serebryannikov, A. E., Ozbay, E. and Vandenbosch, G. A. E. A simple Mie-resonator based meta-array with diverse deflection scenarios enabling multifunctional operation at near-infrared. Nanophoton. 9, 4589-4600 (2020).

19. Luan, J., Yang, S., Liu, D. M. and Zhang, M. M. Polarization and direction controlled asymmetric multifunctional metadevice for focusing, vortex and Bessel beam generation. Opt. Express 28, 3732-3744 (2020).

20. Kwon, H., Arbabi, E., Kamali, S. M., Faraji-Dana, M. S. and Faraon, A. Single-shot quantitative phase gradient microscopy using a system of multifunctional metasurfaces. Nat. Photon. 14, 109-114 (2020).

21. Serebryannikov, A. E., Cakmak, A. O. and Ozbay, E. Multichannel optical diode with unidirectional diffraction relevant total transmission. Opt. Express 20, 14980-14990 (2012).

22. Sell, D., Yang, J., Doshay, S. and Fan, J. A. Periodic dielectric metasurfaces with high-efficiency, multiwavelength functionalities. Adv. Opt. Mat. 5, 1700645 (2017).

23. Vashistha, V., Krawczyk, M., Serebryannikov, A. E. and Vandenbosch, G. A. E. Light guiding, bending, and splitting via local modification of interfaces of a photonic waveguide. Opt. Lett. 44, 4725-4728 (2019).

24. Wang, X., Ding, J., Zheng, B., An, S., Zhai, G. and Zhang, H. Simultaneous realization of anomalous reflection and transmission at two frequencies using bi-functional metasurfaces. Sci. Rep. 8, 1876 (2018).

25. Deng, Z.-L., Cao, Y., Li, X. and Wang, G. P. Multifunctional metasurface: from extraordinary optical transmission to extraordinary optical diffraction in a single structure. Photon. Res. 6, 443-450 (2018).

26. Cai, T., Wang, G.-M., Xu, H.-X., Tang, S.-W., Li, H. and Liang, J.-G. Bifunctional Pancharatnam-Berry Metasurface with High-Efficiency Helicity-Dependent Transmissions and Reflections. Ann. der Physik (Berlin) 530, 1700321 (2017).

27. Cai, T., Tang, S., Wang, G., Xu, H., Sun, S., He, Q. and Zhou, L. High performance bifunctional metasurfaces in transmission and reflection geometries. Adv. Opt. Mat. 5, 1600506 (2017).

28. Wen, D., Yue, F., Li, G. et al. Helicity multiplexed broadband metasurface holograms. Nat. Commun. 6, 8241 (2015).

29. Mutlu, M., Cakmakyapan, S., Serebryannikov, A. E. and Ozbay, E. One-way reciprocal spoof surface plasmons and relevant reversible diodelike beaming. Phys. Rev. B 87, 205123 (2013).

30. Colak, E., Serebryannikov, A. E., Usik, P. V. and Ozbay, E. Diffraction inspired unidirectional and bidirectional beam splitting in defect-containing photonic structures without interface corrugations. J. Appl. Phys. 119, 193108 (2016).

31. Mahmood, N., Kim, I., Mehmood, M. Q., et al. Polarisation insensitive multifunctional metasurfaces based on all-dielectric nanowaveguides. Nanoscale 10, 18323-18330 (2018).

32. Veysi, M., Guclu, C., Boyraz, O. and Capolino, F., Thin anisotropic metasurfaces for simultaneous light focusing and polarization manipulation. J. Opt. Soc. Am. B 32, 318-323 (2015).

33. Ayre, M., Karle, T. J., Wu, L., Davies, T. and Krauss, T. F. Experimental Verification of Numerically Optimized Photonic Crystal Injector, Y-Splitter, and Bend. IEEE J. Selec. Areas Commun. 23, 1390-1395 (2005).

34. Shi, J., Pollard, M. E., Angeles, C. A., Chen, R., Gates, J. C. and Charlton, M. D. B., Photonic crystal and quasi-crystals providing simultaneous light coupling and beam splitting within a low refractive-index slab waveguide. Sci. Rep. 7, 1812 (2017). 
35. Serebryannikov, A. E., Petrov, A. Y. and Ozbay, E. Toward photonic crystal based spatial filters with wide angle ranges of total transmission. Appl. Phys. Lett. 94, 181101 (2009).

36. Staliunas, K. and Sánchez-Morcillo, V. J. Spatial filtering of light by chirped photonic crystals. Phys. Rev. A 79, 053807 (2009).

37. Fehrembach, A.-L., Talneau, A., Boyko, O., Lemarchand, F. and Sentenac, A. Experimental demonstration of a narrowband, angular tolerant, polarization independent, doubly periodic resonant grating filter. Opt. Lett. 32, 2269-2271 (2007).

38. Rabady, R. and Avrutsky, I. Experimental characterization of simultaneous spatial and spectral filtering by an optical resonant filter. Opt. Lett. 29, 605-607 (2004).

39. Liu, W.-N., Chen, R., Shi, W.-Y., Zeng, K.-B., Zhao, F.-L. and Dong, J.-W. Narrow-frequency sharp-angular filters using all-dielectric cascaded meta-gratings. Nanophoton. 9, 3443-3450 (2020).

40. Deng, Z.-L., Zhang, S. and Wang, G. P. Wide-angled off-axis achromatic metasurfaces for visible light. Opt. Express 24, 23118-23128 (2016).

41. Lee, Y. J., Yeo, J., Mittra, R. and Park, W. S. Application of Electromagnetic Band Gap (EBG) superstrates with controllable defects for a class of patch antennas as spatial angular filters. IEEE Trans. Ant. Propag. 53, 224-235 (2005).

42. Tanaka, H., Takai, I., Fujikawa, H. and Iizuka, H. Nearly Polarization-Independent Angular Filters Consisting of OneDimensional Photonic Crystals Realized in the Visible Region. J. Lightwave Technol. 36, 2517-2523 (2018).

43. Qian, Q., Ti, S. and Wang, C. All-dielectric ultra-thin metasurface angular filter. Opt. Lett 44, 3984-3987 (2019).

44. Gawali, S., Gailevičius, D., Garre-Werner, G., Purlys, V., Cojocaru, C., Trull, J., Montiel-Ponsoda, J. and Staliunas, K. Photonic crystal spatial filtering in broad aperture diode laser. Appl. Phys. Lett. 115, 141104 (2019).

45. Piggott, A. Y., Lu, J., Lagoudakis, K. G., Petykiewicz, J., Babinec, T. M. and Vuckovic, J. Inverse design and demonstration of a compact and broadband on-chip wavelength demultiplexer. ACS Photon. 5, 301-305 (2018).

46. Jin, C., Fan, S. Han, S. and Zhang, D. Reflectionless Multichannel Wavelength Demultiplexer in a Transmission Resonator Configuration. IEEE J. Quant. Electron. 39, 160-165 (2003).

47. Matsumoto, T., Fujita, S. and Baba, T. Wavelength demultiplexer consisting of Photonic crystal superprism and superlens. Opt. Express 13, 10768-10776 (2005).

48. Koshiba, M. Wavelength Division Multiplexing and Demultiplexing With Photonic Crystal Waveguide Couplers. J. Lightwave Technol. 19, 1970-1975 (2001).

49. Pahlavan S. and Ahmadi, V. Novel Optical Demultiplexer Design Using Oblique Boundary in Hetero Photonic Crystals. IEEE Photon. Technol. Lett. 29, 511-514 (2017).

50. Yasa, U. G., Turduev, M., Giden, I. H. and Kurt, H. High Extinction Ratio Polarization Beam Splitter Design by LowSymmetric Photonic Crystals. J. Lightwave Technol. 35, 1677-1683 (2017).

51. Shi, Y., Dai, D. and He, S. Proposal for an Ultracompact Polarization-Beam Splitter Based on a Photonic-Crystal-Assisted Multimode Interference Coupler. IEEE Photon. Technol. Lett. 19, 825-827 (2007).

52. Sesay, M., Jin, X. and Ouyang, Z. Design of polarization beam splitter based on coupled rods in a square-lattice photonic crystal. J. Opt. Soc. Am. B 30, 2043-2047 (2013).

53. Slovick, B. A., Zhou, Y., Yu, Z. G., Kravchenko, I. I., Briggs, D. P., Moitra, P., Krishnamurthy, S. and Valentine, J. Metasurface polarization splitter. Phil. Trans. Royal Soc. A375, 20160072 (2017).

54. Ozer, A., Yilmaz, N., Kocer, H., and Kurt, H. Polarization-insensitive beam splitters using all-dielectric phase gradient metasurfaces at visible wavelengths. Opt. Lett. 43, 4350-4353 (2018).

55. Zheng, J., Zhou, C., Feng, J., Cao, H. and Lu, P. Polarizing beam splitter of two-layer dielectric rectangular transmission gratings in Littrow mounting. Opt. Commun. 282, 3069-3075 (2009).

56. Feng, J., Zhou, C., Zheng, J., Cao, H., and Lv, P. Dual-function beam splitter of a subwavelength fused-silica grating. Appl. Opt. 48, 2697-2701 (2009).

57. Ott, D., Divliansky, I., Anderson, B., Venus, G. and Glebov, L. Scaling the spectral beam combining channels in a multiplexed volume Bragg grating. Opt. Express 21, 29620-29627 (2013).

58. Breer, S. and Buse, K. Wavelength demultiplexing with volume phase holograms in photorefractive lithium niobate. Appl. Phys. B 66, 339-345 (1998). 
59. Magath, T. and Serebryannikov, A. E. Fast iterative, coupled-integral-equation technique for inhomogeneous profiled and periodic slabs. J. Opt. Soc. Am. A 22, 2405-2418 (2005).

60. Gumus, M., Giden, I. H. and Kurt, H. Broadband self-collimation in C2 symmetric photonic crystals. Opt. Lett. 43, 2555-2558 (2018).

61. Nojima, S. and Mizoi, T. Photonic band gaps tuned by atomic configurations in binary-compound photonic crystals. Phys. Rev. B 71, 193106 (2005).

62. Noori, M., Soroosh, M. and Baghban, H. Self-Collimation in Photonic Crystals: Applications and Opportunities. Ann. der Physik (Berlin) 530, 1700049 (2018).

63. Lester, M., Skigin, D. C. and Depine, R. A. Control of the diffracted response of wire arrays with double period. Appl. Opt. 47, 1711-1717 (2008).

64. Lester, M., Skigin D. C. and Depine, R. A. Blaze produced by a dual-period array of subwavelength cylinders. J. Opt. A: Pure Appl. Opt. 11, 045705 (2009).

65. Jin, Y. and He, S. Canalization for subwavelength focusing by a slab of dielectric photonic crystal. Phys. Rev. B 75, 195126 (2007).

66. Maigyte, L. and Staliunas, K. Spatial filtering with photonic crystals. Appl. Phys. Rev. 2, 011102 (2015).

67. Arbabi, A., Arbabi, E., Horie, Y., Kamali, S. M. and Faraon, A. Planar metasurface retroreflector. Nat. Photon. 11, 415-420 (2017).

68. Ma, Y. G., Ong, C. K., Tyc, T. and Leonhardt, U. An omnidirectional retroreflector based on the transmutation of dielectric singularities. Nat. Mat. 8, 639-642 (2009).

69. Wang, Q., Zhou, L., Wan, P., et al. Retroreflection from a single layer of electromagnetic Helmholtz cavities based on magnetic symmetric dipole modes, Opt. Express 28, 30606-30615 (2020).

70. Beaky, M. M., Burk, J. B., Everitt, H. O., Haider, M. A. and Venakides, S. Two-Dimensional Photonic Crystal Fabry-Perot Resonators with Lossy Dielectrics. IEEE Trans. Microwave Theory Tech. 47, 2085-2091 (1999).

71. Chen, X., Chardin, C., Makles, K., Caër, C., Chua, S., Braive, R., Robert-Philip, I., Briant, T., Cohadon, P.-F., Heidmann, A., Jacqmin, T. and Deléglise, S. High-finesse Fabry-Perot cavities with bidimensional Si3N4 photonic-crystal slabs. Light Sci. Appl. 6, e16190 (2017).

72. Petit, R., Ed., Electromagnetic Theory of Gratings, Springer: Berlin Heidelberg, 1980.

73. Busch, K., Lölkes, S., Wehrspohn, R. B. and Föll, H., Eds., Photonic Crystals: Advances in Design, Fabrication, and Characterization, Wiley, 2006.

74. Inoue, K. and Ohtaka K., Eds., Photonic Crystals: Physics, Fabrication and Applications, Springer: Berlin Heidelberg, 2004.

\section{Author contributions statement}

A. E. S. and D. C. S. suggested the concept. A. E. S. performed simulations. D. C. S., E. O., and G. A. E. V. assisted by analyzing results. All authors contributed to the formulation of the goals of the study and reviewed the manuscript.

\section{Additional information}

The authors declare no competing financial interests. 